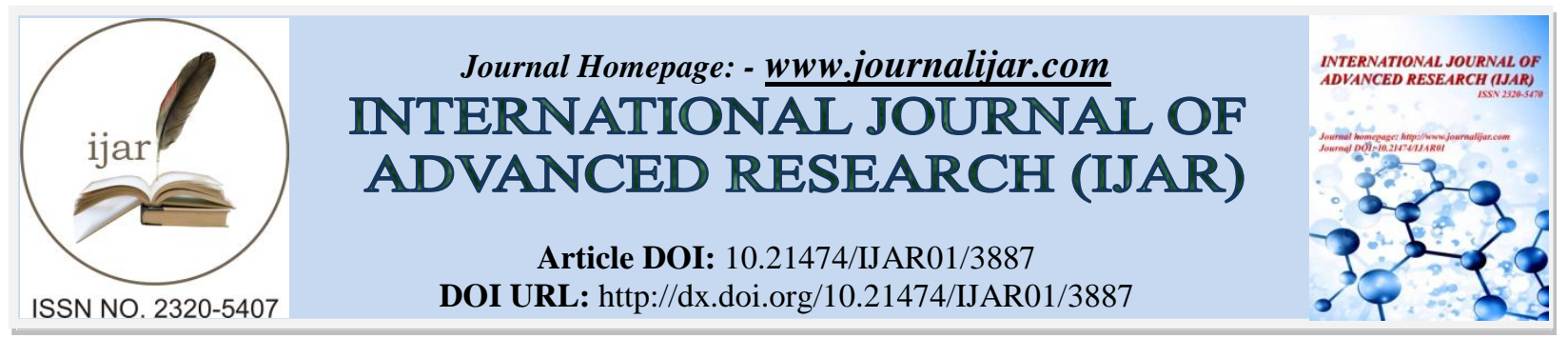

RESEARCH ARTICLE

\title{
RENAL PERINEPHRIC ABSCESS COMPLICATED WITH PSOAS MUSCLE ABSCESS (CASE REPORT)
}

\author{
Didbaridze $T^{1}$, Kochiashvili $D^{2}$, Kvakhajelidze $V^{3}$, Kochiashvili $\mathbf{G}^{3}$,Gogokhia $\mathrm{N}^{\mathbf{5}}$ and Mikaberidze $\mathrm{K}^{\mathbf{6}}$. \\ 1. Microbiologist MD.PhD. TSMU The First University Clinic(Tbilisi,Georgia). \\ 2. Head of Department of Urology, TSMU Professor.MD.PhD(Tbilisi,Georgia). \\ 3. Urologist.TSMU Department of Urology(Tbilisi,Georgia). \\ 4. TSMU Department of Urology. Assistant professor. MD.PhD(Tbilisi,Georgia). \\ 5. Head of Clinical laboratory MD.PhD. TSMU the First University Clinic (Tbilisi,Georgia). \\ 6. Laboratory Physician. TSMU the First University Clinic(Tbilisi, Georgia).
}

\section{Manuscript Info}

Manuscript History

Received: 08 February 2017

Final Accepted: 07 March 2017

Published: April 2017

Key words:-

Proteus mirabilis, psoas abscess, perinephric abscess

\begin{abstract}
Perinephric abscess is an uncommon complication of urinary tract infections. The incidence ranges from 1-10 cases for every 10000 hospital admissions. Men and women are affected with equal frequency.E.coli, Proteus speacies and Staphylococcus aureus are the unusual etiologic organisms.

There were very few reports about urinary tract infections such as renal perinephric abscess complicated with psoas muscle abscess. Renal and perirenal abscess can complicate a urologic infection(usually due to gram-negative enteric bacilli or a polymicrobial infection). This clinical case of a 24 year old men with predisposing condition for secondary psoas muscle abscess $(6$ year history of the right non functional kidney), is interesting because of unusual etiologic organisms for psoas muscle abscess Proteus mirabilis, which is highly flagellate, have stone formation ability which is very difficult to clear with only antibiotics, has predilection for the kidney and are difficult to eradicate.
\end{abstract}

Copy Right, IJAR, 2017,. All rights reserved.

\section{Introduction:-}

Perinephric abscess is an uncommon complication of urinary tract infections. The incidence ranges from 1-10 cases for every 10000 hospital admissions. Men and women are affected with equal frequency.E.coli, Proteus speacies and Staphylococcus aureus are the unusual etiologic organisms(1,2,3).

There were very few reports about urinary tract infections such as renal perinephric abscess complicated with psoas muscle abscess. Renal and perirenal abscess can complicate a urologic infection(usually due to gram-negative enteric bacilli or a polymicrobial infection). As an example,renal abscess occurs more frequently than perinephric abscess. Perinephric abscess consists of a more diffuse liquefaction located between the renal capsule and Gerota's fascia. While infecting the urinary tract ,Proteus mirabilis has a predilection for the kidney.Finally and importantly, not only does this bacterium cystitis and acute pyelonephritis, but the production of urinary stones, a hallmark of infection with this organism(4,5). 
P. mirabilis, a motile gram-negative bacterium that differentiates from a short vegetative rod to an elongated, highly flagellated form, is found in soil, water, and the human intestinal tract .Stone formation is caused by the expression of a highly active urease that hydrolyzes urea to ammonia, causing local $\mathrm{pH}$ to rise with subsequent precipitation of magnesium ammonium phosphate (struvite) and calcium phosphate (apatite) crystals . The stones resulting from aggregation of such crystals complicate infection for three reasons. First, the P.mirabilis caught within the interstices of the forming stones are very difficult to clear with only antibiotics. Second, the stone is a nidus for nonP.mirabilis bacteria to establish UTI that also are difficult to eradicate. Third, the stone can obstruct urine flow; pelvic and renal stones are often associated with acute pyelonephritis, pyonephrosis, and/or chronic pyelonephritis $(6,7,8)$.

Predisposing conditions for secondary psoas muscle abscess include diabetes, immunosupression, and renal feilure. Secondary psoas abscess is usually caused by enteric bacteria:E.coli, Streptococcus species, Enterobacter and Salmonella enteritidis.Adjustment should be based on report of abscess fluid culture and sensitivity testing $(9,10,11)$.

\section{Clinical Case Report:-}

We present the case of a 24 year old men with 6 year history of the right non functional kidney, who presented in TSMU The First University clinic with fatigue, fever $39^{\circ} \mathrm{C}$ and chills. 2 month before he had episode of severe right side back pain(posterior lumbal region) and was consulted with urologist.MRI of abdomen was done and biopsy was suggestion. During week severity of pain was decrease,however fatigue and hectic fever still was existed. Healthy condicion was worsed and he visited ambulatory to our clinic. Following laboratory analyses was done:Whole blood analysis, urine test, creatinine in blood.CT angiography of the abdomen and pelvic revealed right side perinephric abscess disseminated to retroperitoneal, psoas muscle and subdiaphragmatic $8^{\text {th }}-11^{\text {th }}$ rib level anterior and posterior axillary line proation and right side kidney stones $0,6 \mathrm{~cm}$. Right kidney excretory function wasn't observed.

Surgery involved:Right side lumbotomy, nephrectomy, psoas muscle fasciotomy and drainage of the abscess. Irrigated tube was placed in psoas muscule. Abscess fluid was taken and sent under compliance with the appropriate protocol for culture and sensitivity test.The research included: isolation of a pure culture, Gram staining, use of the rapid identification systems (api20E,api Staph, api Strep, api A, api20Caux, biomerieux) and Antimicrobial Susceptibility Testing (AST) determination through Kirby-Bauer method by using of standard discs (EUCAST guidelines). Abscess fluid was cultured in aerobic and anaerobic atmosphere (Gen-Bag biomerieux) on the enrichment and differential-diagnostic medium. After 18-24 hours of incubation at $37^{\circ} \mathrm{C}$, appeared colony with swarming growth on the bloody agar (TSA 5\% with sheep blood) and on Endo agar (for Enterobacteriaceae family) which were stained by use of Gram procedure and bacteria were identified by the amplification profile index special panel (api20E), identification of the bacteria was determined by Apiweb. The isoaited was a Gramnegative, facultatively anaerobic,rod-shaped bacterium Proteus mirabilis $10^{8} / \mathrm{ml}(\mathrm{CFU} / \mathrm{ml})$.

Cytology study of right kidney tissues revealed:nephrosclerosy, renal parenchymal atrophy and chronic inflammatory changes.

Postoperative treatment was started with ceftriaxone along with metronidazole, which based on the local susceptibility testing was continued and irrigated tube was washed with betadine solution.Patient was discharge after week of admission in hospital. Sutures were removed after 3 days from discharge. Patient health condicion was setisfide.

\section{Conclusion:-}

This clinical case is interesting because perinephric abscess is an uncommon complication of urinary tract infections and very few reports are about urinary tract infections such as renal perinephric abscess complicated with psoas muscle abscess caused by unusual etiologic organisms Proteus mirabilis, which is highly flagellate and have stone formation ability . 


\section{Reference:-}

1. Lee SH, Jung HJ, Mah SY, Chung BH. Renal abscesses measuring $5 \mathrm{~cm}$ or less: outcome of medical treatment without therapeutic drainage. Yonsei Med J 2010; 51:569.

2. Hsu RB, Lin FY. Psoas abscess in patients with an infected aortic aneurysm. J Vasc Surg 2007; 46:230. Ijaz M, Sakam S, Ashraf U, Marquez JG. Unusual Presentation of Recurrent Pyogenic Bilateral Psoas Abscess Causing Bilateral Pulmonary Embolism by Iliac Vein Compression. Am J Case Rep 2015; 16:606.

3. Volpin A, Kini SG, Berizzi A. Psoas muscle pyogenic abscess in association with infected hip arthroplasty: a rare case of simultaneous bilateral presentation. BMJ Case Rep 2015; 2015.

4. Zissin R, Gayer G, Kots E, et al. Iliopsoas abscess: a report of 24 patients diagnosed by CT. Abdom Imaging 2012; 26:533.

5. Takada T, Terada K, Kajiwara H, Ohira Y. Limitations of using imaging diagnosis for psoas abscess in its early stage. Intern Med 2015; 54:2589.Lee JK, Glazer HS. Psoas muscle disorders: MR imaging. Radiology 1986; 160:683.

6. Kao PF, Tzen KY, Tsui KH, et al. The specific gallium-67 scan uptake pattern in psoas abscesses. Eur J Nucl Med 2016; 25:1442.

7. Hsieh MS, Huang SC, Loh el-W, et al. Features and treatment modality of iliopsoas abscess and its outcome: a year hospital-based study. BMC Infect Dis 2013; 13:578.

8. Buttaro M, González Della Valle A, Piccaluga F. Psoas abscess associated with infected total hip arthroplasty. J Arthroplasty 2013; 17:230.

9. Lee BB, Ngan Kee WD, Griffith JF. Vertebral osteomyelitis and psoas abscess occurring after obstetric epidural anesthesia. Reg Anesth Pain Med 2012; 27:220.

10. Dolfin D, Barkin J, Arenson AM, Herschorn S. Psoas abscess after operation on lumbar spine. Urology 2015; 21:544

11. Lin SS, Vaccaro AR, Reisch S, et al. Low-velocity gunshot wounds to the spine with an associated transperitoneal injury. J Spinal Disord 2014; 8:136. 